\title{
Klaus Herbers, Harald Müller (dir.), Lotharingien und das Papsttum im Früh- und Hochmittelalter
}

\section{Tristan Martine}

\section{OpenEdition}

\section{Journals}

Édition électronique

URL : http://journals.openedition.org/ifha/10054

DOI : 10.4000/ifha. 10054

ISSN : 2198-8943

Éditeur

IFRA - Institut franco-allemand (sciences historiques et sociales)

Référence électronique

Tristan Martine, « Klaus Herbers, Harald Müller (dir.), Lotharingien und das Papsttum im Früh- und Hochmittelalter », Revue de l'IFHA [En ligne], Date de recension, mis en ligne le 18 avril 2019, consulté le 24 septembre 2020. URL : http://journals.openedition.org/ifha/10054 ; DOI : https://doi.org/10.4000/ ifha. 10054

Ce document a été généré automatiquement le 24 septembre 2020.

(C)IFHA 


\title{
Klaus Herbers, Harald Müller (dir.), Lotharingien und das Papsttum im Früh- und Hochmittelalter
}

\author{
Tristan Martine
}

\section{RÉFÉRENCE}

Klaus Herbers, Harald Müller (dir.), Lotharingien und das Papsttum im Früh- und Hochmittelalter. Wechselwirkungen im Grenzraum zwischen Germania und Gallia, Berlin/ Boston : De Gruyter, (Abhandlungen der Akademie der Wissenschaften zu Göttingen. Neue Folge 45), 2017, VIII + 268 p., $99,95 €$ 
Le volume s'ouvre par un rapide Vorwort, dans lequel Klaus Herbers et Harald Müller reviennent sur la particularité de la Lotharingie comme « espace de l'entredeux ", notamment du point de vue des éditions diplomatiques. En effet, le «Göttinger Papsturkunden-Werk », a pour objectif, depuis 1896, d'établir une édition critique des actes pontificaux antérieurs à Innocent III (1198-1216), qui sont à recenser dans les archives de leurs destinataires, alors qu'à partir du pontificat de ce dernier pape, les registres pontificaux nous sont parvenus. Le travail a alors été conçu en trois étapes clairement distinctes: recenser touts les documents pontificaux, puis en publier les regestes, et enfin en proposer une édition critique. Le travail fut réparti par espace géographique: aux entreprises plus

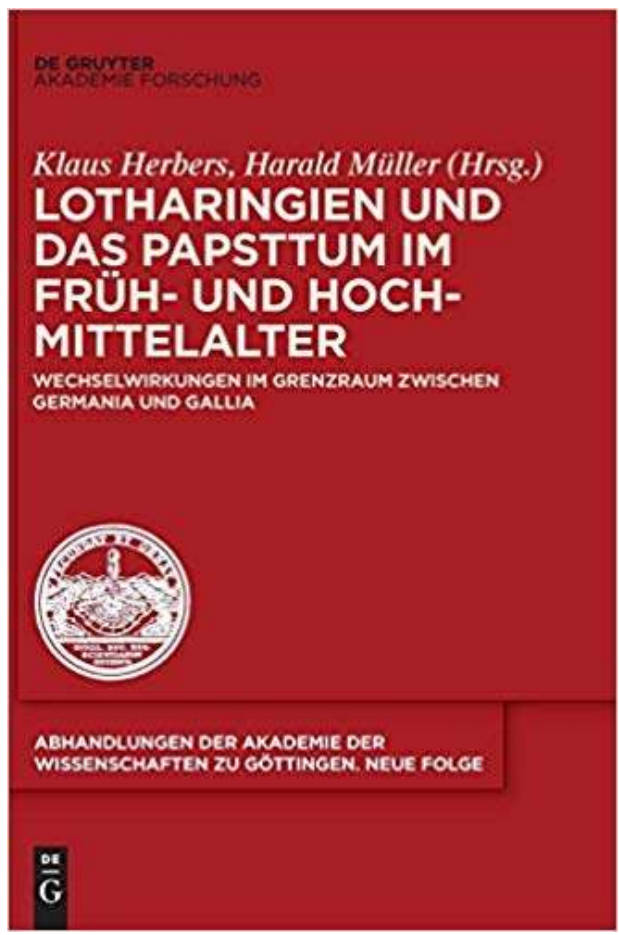
anciennes de l' «Italia Pontificia » et de la «Germania Pontificia » s'est ajoutée récemment la «Gallia Pontificia », projet à long terme porté par l'Institut Historique Allemand de Paris en coopération avec l'École nationale des chartes, et qui concerne donc l'espace français.

Où placer la Lotharingie dans ces différentes entreprises? La logique voudrait bien sûr que l'on intègre les diocèses de Liège ou de Metz dans la «Germania Pontificia », et pourtant cela aurait choqué plus d'un Français de voir les actes verdunois édités dans une édition englobant les actes allemands. Pour des raisons liées à la géopolitique contemporaine, cet ancien espace central de l'espace carolingien est resté en marge des différentes entreprises nationales d'édition des actes pontificaux. Un colloque s'est donc tenu du 17 au 20 septembre 2014 à Aix-la-Chapelle, lieu symbolique s'il en est pour la Lotharingie. L'objectif était d'étudier les interactions et les relations entre la Lotharingie et la papauté dans un espace frontalier entre Germania et Gallia. Cette manifestation scientifique, et les actes qui en découlent, doivent en fait être compris comme un "plaidoyer» (l'expression est utilisée par K. Herbers) pour l'élaboration d'une « Lotharingia Pontificia ».

L'ensemble des articles est réparti en deux parties : la première est consacrée à la fois aux personnes et aux espaces. K. Herbers revient tout d'abord sur l'histoire du «Göttinger Papsturkunden-Werk», puis il fait un rapide bilan du matériau diplomatique conservé par les fondations ecclésiastiques lotharingiennes, des éditions et des inventaires déjà existants. Bien davantage que dans le diocèse de Liège, il semble nécessaire de continuer des investigations détaillées dans les diocèses de Metz, Toul et Verdun. Il insiste également sur des aspects méthodologiques, dans l'objectif d'intégrer pleinement les futures "Lotharingia Pontificia» dans une banque de données entièrement compatible avec celle des «Germania », « Bohemia », « Iberia » et " Gallia Pontificia ». 
M. Margue s'intéresse ensuite à la Lotharingie comme espace de réformes, du $\mathrm{X}^{\mathrm{e}}$ au début $\mathrm{du} \mathrm{XII}^{\mathrm{e}}$ siècle, en interrogeant l'idée communément admise selon laquelle la Lotharingie aurait été une terre réformatrice au $\mathrm{X}^{\mathrm{e}}$ siècle (avec la réforme de Gorze notamment), et que ce milieu réformateur aurait imprégné Léon IX et son entourage, et donc, in fine, suscité la réforme grégorienne. Il interroge notamment l'idée, que l'on retrouve par exemple dans les travaux de M. Parisse, d'une « influence lotharingienne dans l'évolution de la politique pontificale » et d'un "axe lotharingien » qui serait un lieu privilégié pour le "renouvellement des idées et des hommes ». Il fait tout d'abord le point sur la question d'une identité propre à l'espace lotharingien, ainsi sur celle du concept de "réforme ", en intégrant notamment dans la réflexion le modèle clunisien et les travaux récents de $\mathrm{S}$. Vanderputten qui montrent la nécessité de se méfier d'une approche systémique des réformes qui partirait du postulat de réformes organisées à partir d'un centre unique, alors qu'en réalité, ces «mouvements » doivent avant tout être compris à l'échelon de chaque institution religieuse, en prenant en compte le rôle de l'abbé, d'une part, et le contexte d'écriture de l'histoire de ces réformes, d'autre part. Surtout, la recherche récente a montré la nécessité de distinguer clairement les mouvements réformateurs lotharingiens $\mathrm{du} \mathrm{X}^{\mathrm{e}}$ siècle de la réforme grégorienne: l'évêque Brunon de Toul a mené dans son diocèse une politique s'inscrivant pleinement dans celle des évêques d'Empire. Il faut également se départir d'une vision manichéenne qui opposerait des groupes grégoriens contre des groupes favorables à l'empereur, alors qu'une étude de détail montre la complexité des situations locales, loin des approches théorique et systématique surplombantes, qui feraient de la Lotharingie un espace réformateur par essence.

M. Kerner revient ensuite sur les liens entre Aix-la-Chapelle et Rome, de Charlemagne à Lothaire II, à partir d'exemples précis comme celui des conflits entre Nicolas $\mathrm{I}^{\text {er }}$ et l'épiscopat lotharingien. R. Schieffer étudie les voyages des papes en Lotharingie, du début du IX ${ }^{\mathrm{e}}$ au milieu du XII ${ }^{\mathrm{e}}$ siècle, révélateurs de leur politique et de leur puissance : ainsi leur absence dans l'espace lotharingien tout au long du $\mathrm{X}^{\mathrm{e}}$ siècle est significative, tandis qu'à l'inverse, à partir de Léon IX, cette région frontalière est très fréquemment parcourue par les évêques de Rome. À l'inverse, K. A. Frech s'intéresse aux Lotharingiens présents à Rome à l'époque des cinq papes « allemands » du milieu du XI ${ }^{\mathrm{e}}$ siècle, de Clément II (1046-1047) à Étienne IX (1057-1058) : il analyse ainsi la présence de Lotharingiens dans des synodes romains, d'une part, à la curie, d'autre part, tout en s'intéressant aux contingents militaires recrutés dans l'espace lotharingien. Il montre surtout le rôle central joué dans ces différents mouvements par la figure de Léon IX (1049-1054). Enfin, J.-L. Kupper étudie les relations religieuses et politiques entre le diocèse de Liège et la papauté $d u \mathrm{X}^{\mathrm{e}}$ au XII ${ }^{\mathrm{e}}$ siècle, en prenant en considération à la fois les séjours romains de clercs liégeois, l'apparition à Liège de légats pontificaux ou du pape lui-même, ainsi que l'octroi de bulles pontificales aux diocésains liégeois et aux différentes institutions du diocèse. Il montre l'enjeu stratégique que constituait ce diocèse qui, de fleuron de l'Église impériale, devint progressivement et cela jusqu'au début du XIII ${ }^{e}$ siècle, une Église pontificale.

La seconde partie du volume est dédiée à l'étude des sources (diplomatiques, épistolaires et canoniques) et de leur transmission. D. Berger consacre une étude détaillée et érudite à ce même diocèse liégeois comme destinataire d'actes pontificaux, principalement aux $\mathrm{XI}^{\mathrm{e}}$ et $\mathrm{XII}^{\mathrm{e}}$ siècles, en comprenant ces derniers comme le fruit d'interactions entre les deux parties, le destinataire et la papauté, en amont de la rédaction et en montrant également l'état encore insatisfaisant de nos connaissances 
sur ces sources. À sa suite, J.-B. Renault interroge l'influence des privilèges pontificaux sur les actes des évêques de Metz au XII ${ }^{\mathrm{e}}$ siècle, et notamment la question de l'accès des rédacteurs aux modèles, d'un point de vue pratique : les rédacteurs d'actes épiscopaux ont consulté les documents pontificaux conservés dans les chapitres des institutions monastique et canoniale, en ont conservé des copies, constituant ainsi un répertoire de formules. Cette imitation des formules pontificales se trouve à partir des années 1120 en Lorraine, c'est-à-dire plus tardivement que la province de Reims voisine, ce que le positionnent politique des évêques dans ce contexte grégorien pourrait expliquer, tandis que l'arrivée d'Etienne de Bar sur le siège messin coïncide avec un véritable souci d'affirmation de l'évêque, qui s'exprime par le biais de ces évolutions diplomatiques.

B.-M. Tock analyse ensuite la permanence et l'évolution des formules des privilèges pontificaux au $\mathrm{XII}^{\mathrm{e}}$ siècle, à partir de 45 privilèges de confirmation générale reçus par des abbayes cisterciennes de la province de Reims. Il montre ainsi que le formulaire utilisé par la chancellerie pontificale n'était pas rigide au point qu'il soit superflu d'en éditer les formules: les protocoles et les formules finales témoignent d'une certaine instabilité (ce qui, au passage, justifie le fait que les éditions reproduisent désormais le texte intégral de ces actes, jusque dans leurs moindres détails). Cela s'explique non seulement par le fait que la chancellerie fasse évoluer son formulaire de manière cohérente, en fonction d'une certaine chronologie, avec des tâtonnements dans les années 1130-1140, mais aussi par le rôle de l'impétrant, qui guide manifestement un peu l'élaboration du privilège.

$M$. Witzleb envisage ensuite un autre type de sources, la documentation épistolaire, afin de montrer leur importance pour l'histoire de l'espace lotharingien, à partir de l'exemple des diocèses de Liège, de Verdun, de Metz et de Toul jusque vers 1200. Alors que cette région n'est pas réputée pour la quantité de sa documentation épistolaire, $\mathrm{M}$. Witzleb montre certes, diocèse par diocèse, leur relative faiblesse quantitative, mais il souligne également leur importance, à partir par exemple de la correspondance de Rathier de Vérone, qui fut évêque de Liège d'où il était originaire. L.Kéry consacre, quant à elle, un article très détaillé aux collections canoniques jusqu'au milieu du XII siècle, dans lequel elle montre, à l'aide de l'étude de plusieurs manuscrits dont l'origine lotharingienne pose problème, qu'à l'exception de deux œuvres très importantes, celle de Réginon de Prüm ainsi que le Liber de misericordia et iustitia d'Alger de Liège, il n'y a aucune preuve convaincante d'une activité canonique importante en Lotharingie. J. Dahlaus s'intéresse quant à lui à la transmission de ces documents à l'époque moderne et à la manière dont la plupart d'entre eux sont, du XVI ${ }^{e}$ au XIX siècle, arrivés dans des fonds d'archives à Paris.

Pour finir, H. Müller propose, en guise de remarques conclusives, un bilan des particularités propres à l'espace lotharingien, dont la caractéristique première est de se trouver "zwischen den Reichen" (p. 244). Il souligne la faiblesse non seulement des recensions et des éditions des actes pontificaux pour cette zone, mais aussi des études portant sur les relations entre Rome et cette région. Tout en interrogeant la question d'une identité lotharingienne, il semble définitivement pertinent de reprendre la question des rapports de la Lotharingie avec la papauté à l'aide d'une nouvelle étude des sources disponibles, ce qui permettrait de mieux comprendre cette zone de contact, véritable « regio mixta » (p. 253) entre France et Allemagne. 
Ce volume remplit parfaitement la tâche qui lui avait été attribuée par K. Herbers : il convainc entièrement du bien-fondé et de l'utilité heuristique d'un projet de "Lotharingia Pontificia», dans cet espace que la plupart des communications considèrent comme étant pour l'Empire un "kirchliche und kulturelle Kernlandschaft " (p. 123). Inventaires, regestes et édition de cette documentation pontificale pourraient s'avérer extrêmement utiles. Une étude détaillée des actes pontificaux, dans leur entièreté, jusqu'aux formules habituellement considérées comme les plus usuelles et uniformes, semble nécessaire, non seulement pour aider à la datation et au dépistage des faux, mais aussi pour mieux comprendre le fonctionnement de la chancellerie et ses relations avec ses clients et usagers (p. 175). Mais au-delà, la diversité des approches contenues dans ce recueil montre bien la richesse des travaux possibles à partir cette documentation encore trop peu étudiée pour cet espace de l'entre-deux.

\section{INDEX}

Index chronologique : Moyen Âge

Thèmes : Histoire des villes et des régions, Histoire religieuse 